\title{
POLIPOSE GASTRODUODENAL EM DOENTES COM POLIPOSE ADENOMATOSA FAMILIAR PÓS-RETOCOLECTOMIA
}

\author{
Raquel Franco LEAL, Maria de Lourdes Setsuko AYRIZONO, Cláudio Saddy Rodrigues COY, \\ Francisco CALLEJAS-NETO, João José FAGUNDES e Juvenal Ricardo Navarro GÓES
}

\begin{abstract}
RESUMO - Racional - As manifestações extracólicas, como os pólipos gastroduodenais e o tumor do duodeno, são fatores que influenciam a morbimortalidade dos doentes com polipose adenomatosa familiar no seguimento pós-retocolectomia total. Objetivo - Investigar a freqüência destas alterações em doentes com polipose adenomatosa familiar e verificar a eficácia do rastreamento endoscópico. Métodos - No período de 1984 a 2005, 62 doentes com polipose adenomatosa familiar pós-retocolectomia foram estudados retrospectivamente pelo Grupo de Coloproctologia da Faculdade de Ciências Médicas da Universidade Estadual de Campinas, SP. O tempo de seguimento médio pós-operatório foi de 81,9 meses, sendo que em 53 (85,5\%) foi possível analisar a ocorrência de pólipos gastroduodenais. Resultados - Dos 53 doentes em seguimento, 27 (50,9\%) apresentavam pólipos gastroduodenais. Em 8 (15,4\%) os pólipos adenomatosos eram gástricos, 14 (27\%) pólipos duodenais e $5(9,6 \%)$ pólipos gástricos e duodenais. Dois doentes (3,8\%) desenvolveram adenoma duodenal com displasia de alto grau. E outro $(1,9 \%)$, adenocarcinoma em papila duodenal. Conclusão - O rastreamento endoscópico, desta forma, é de grande importância e o objetivo é detectar, o mais precocemente possível, os casos de adenocarcinoma duodenal e pólipos gastroduodenais com displasia de alto grau.
\end{abstract}

DESCRITORES - Polipose adenomatosa do colo. Pólipos intestinais. Bolsas do colo. Proctocolectomia restauradora.

\section{INTRODUÇÃO}

A polipose adenomatosa familiar (PAF) é doença autossômica dominante que afeta de 1 a 8 indivíduos a cada 10.000 habitantes, distribuídos igualmente em ambos os sexos e presente em todos os grupos étnicos. Geralmente o aparecimento dos pólipos ocorre na puberdade, sendo caracterizada pela presença de mais de 100 pólipos adenomatosos no cólon e reto ${ }^{(7,14,17)}$.

Mesmo após a retocolectomia, a morbimortalidade é influenciada pelas manifestações extracólicas, como os tumores desmóides, pólipos gastroduodenais e os tumores duodenais, sendo os tumores desmóides e duodenais as principais causas de óbito pós-retocolectomia ${ }^{(8)}$.

O rastreamento endoscópico pós-operatório para pesquisa de pólipos gastroduodenais tem mostrado incidência de $90 \%$ a $95 \%$ de pólipos, sendo que 5\% desenvolvem câncer ${ }^{(4,5,10)}$. No entanto, este risco é 100 a 300 vezes maior que na população em geral, na qual o carcinoma duodenal é de ocorrência $\operatorname{rara}^{(3,11,12)}$. Os achados endoscópicos muitas vezes geram dúvidas a respeito das condutas a serem realizadas, não estando suas freqüências bem estabelecidas na literatura ${ }^{(6)}$.

O objetivo deste estudo foi investigar a freqüência de pólipos gastroduodenais na população de doentes com PAF em seguimento de retocolectomia em hospital universitário, a ocorrência de neoplasia local decorrente da transformação maligna desses pólipos e o grau de aderência ao esquema de acompanhamento pós-operatório.

\section{MÉTODOS}

Trata-se de estudo retrospectivo, descritivo, a partir da análise de prontuários. Entre 1984 a 2005, 62 doentes com PAF foram submetidos a retocolectomia, pelo Grupo de Coloproctologia do Hospital Clinicas da Universidade Estadual de Campinas, SP.

Trinta e cinco $(56,5 \%)$ eram do sexo feminino, com média de idade de 29,1 (13-65) anos. Dez (16,1\%) já apresentavam adenocarcinoma do cólon/reto no momento da cirurgia. Cinqüenta e quatro doentes $(85,5 \%)$ foram submetidos a retocolectomia com reservatório ileal e oito $(14,5 \%)$ a retocolectomia com anastomose ileoretal. Nas cirurgias de reservatório ileal foram realizadas mucosectomia e anastomose ileoanal manual.

Os critérios de inclusão foram doentes com polipose adenomatosa familiar em seguimento pós-operatório e realizando regularmente o acompanhamento endoscópico. Foram excluídos os que perderam seguimento ou que evoluíram para o óbito.

O tempo médio de pós-operatório foi de 81,9 (2-264) meses, sendo que sete doentes perderam seguimento, sendo

Grupo de Coloproctologia, Disciplina de Moléstias do Aparelho Digestório, Faculdade de Ciências Médicas, Universidade Estadual de Campinas, SP.

Correspondência: Dr. Juvenal Ricardo Navarro Góes - Rua Dr. Antonio Augusto de Almeida, 37 - Cidade Universitária - 13083-755 - Campinas, SP. E-mail: rgoes@mpcnet.com.br 
excluídos da análise. Ocorreram três óbitos, um doente no pósoperatório recente ainda no início da casuística, outro no $6^{0}$ mês de pós-operatório por tumor cerebelar e um no pós-operatório tardio por abdome agudo obstrutivo e perfurativo há 3 meses. Este último não possuía pólipos gastroduodenais até esta data e foi incluído no estudo, uma vez que participou regularmente do seguimento pós-operatório até apresentar a intercorrência referida.

No seguimento, esses doentes foram regularmente submetidos a endoscopia digestiva alta (EDA), sendo que a freqüência dos exames foi estabelecida como sendo anual nos doentes com pólipos gastroduodenais e nos demais, a cada 3 anos. Os dados referem-se à última EDA realizada. Os pólipos encontrados foram retirados por via endoscópica e enviados para exame anatomopatológico.

\section{RESULTADOS}

Dos 53 doentes com PAF em seguimento pós-operatório de retocolectomia, $27(50,9 \%)$ apresentavam adenomas gastroduodenais. Oito $(15,4 \%)$ possuíam somente pólipo gástricos, $14(27 \%)$ somente pólipos duodenais e $5(9,6 \%)$, pólipos gástricos e duodenais.

Dos 27 doentes que possuíam pólipos, $18(66,6 \%)$ eram do sexo feminino, com média de idade de 32 (18-76) anos. Dentre os 19 (36,5\%) com pólipos duodenais, uma paciente com 28 anos de idade, apresentou duodeno repleto de pólipos pequenos, com biopsias aleatórias, mostrando adenoma com displasia de alto grau, sendo submetida a duodenectomia com preservação pancreática e reinserção da papila na alça jejunal. A doente encontra-se no $6^{0}$ mês de pós-operatório com boa evolução. Outra doente de 31 anos apresentou pólipo em papila duodenal com displasia de alto grau, sendo submetida a duodenectomia, ressecção e reimplante da papila duodenal, evoluindo bem no $6^{0}$ mês de pós-operatório (Figura 1).

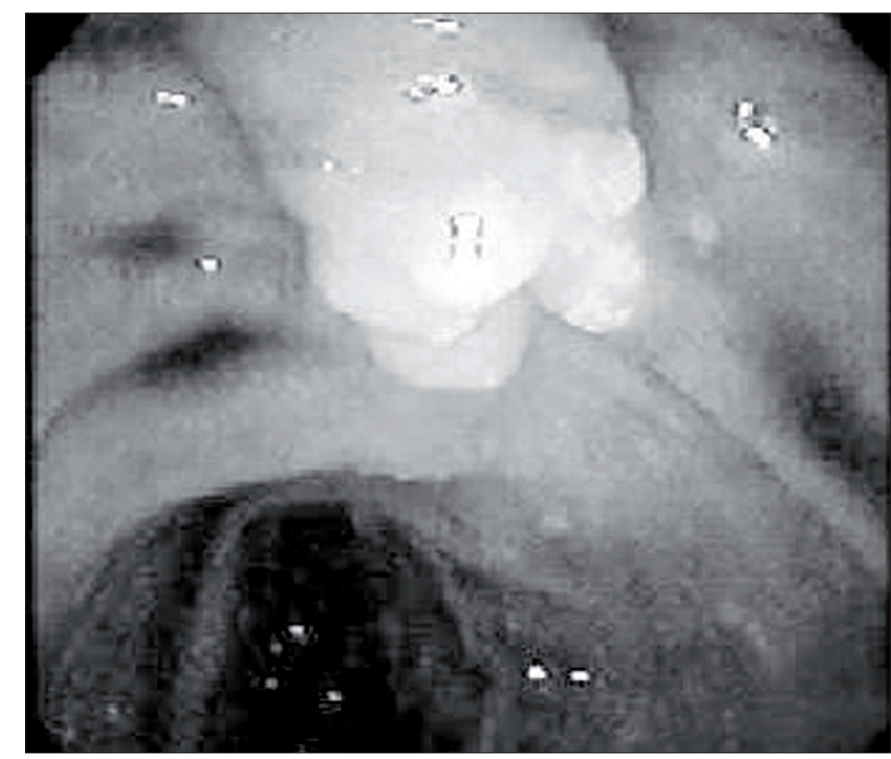

FIGURA 1. Duodenoscopia com adenoma duodenal

TABELA 1. Pólipos gastroduodenais em doentes com PAF pós-retocolectomia

\begin{tabular}{|c|c|c|c|c|c|c|}
\hline Doente & Sexo & $\begin{array}{l}\text { Idade } \\
\text { (anos) }\end{array}$ & $\begin{array}{l}\text { Tempo de seguimento pós- } \\
\text { proctocolectomia (meses) }\end{array}$ & $\begin{array}{c}\text { Localização dos } \\
\text { pólipos gastroduodenais }\end{array}$ & Anátomopatológico & Conduta \\
\hline 1 & $\mathrm{~F}$ & 35 & 264 & Estômago & $*$ & ** \\
\hline 2 & M & 60 & 240 & Estômago & $*$ & $* *$ \\
\hline 3 & $\mathrm{~F}$ & 40 & 192 & Estômago & $*$ & $* *$ \\
\hline 4 & M & 36 & 180 & Estômago & $*$ & $* *$ \\
\hline 5 & M & 38 & 96 & Duodeno & $*$ & $* *$ \\
\hline 6 & $\mathrm{~F}$ & 28 & 48 & Duodeno & Adenoma c/ displasia de alto grau & $\begin{array}{l}\text { Duodenectomia com } \\
\text { preservação pancreática }\end{array}$ \\
\hline 7 & $\mathrm{M}$ & 21 & 48 & Duodeno & $*$ & $* *$ \\
\hline 8 & M & 20 & 72 & Estômago & $*$ & $* *$ \\
\hline 9 & $\mathrm{~F}$ & 36 & 60 & Duodeno & Adenoma c/ displasia de alto grau & $\begin{array}{l}\text { Duodenotomia, ressecção e } \\
\text { reimplante da papila }\end{array}$ \\
\hline 10 & $\mathrm{~F}$ & 23 & 36 & Duodeno & $*$ & $* *$ \\
\hline 11 & $\mathrm{~F}$ & 76 & 108 & Estômago/duodeno & Adenocarcinoma papila duodenal & $\begin{array}{l}\text { Duodenotomia, ressecção e } \\
\text { reimplante da papila }\end{array}$ \\
\hline 12 & $\mathrm{~F}$ & 32 & 60 & Duodeno & $*$ & $* *$ \\
\hline 13 & $\mathrm{~F}$ & 28 & 60 & Duodeno & $*$ & $* *$ \\
\hline 14 & $\mathrm{~F}$ & 32 & 52 & Estômago/duodeno & $*$ & $* *$ \\
\hline 15 & $\mathrm{~F}$ & 37 & 44 & Estômago & $*$ & $* *$ \\
\hline 16 & $\mathrm{~F}$ & 41 & 48 & Duodeno & $*$ & $* *$ \\
\hline 17 & $\mathrm{~F}$ & 23 & 60 & Estômago & $*$ & $* *$ \\
\hline 18 & $\mathrm{M}$ & 27 & 48 & Duodeno & $*$ & $* *$ \\
\hline 19 & M & 21 & 48 & Duodeno & $*$ & $* *$ \\
\hline 20 & $\mathrm{~F}$ & 27 & 36 & Duodeno & $*$ & $* *$ \\
\hline 21 & $\mathrm{~F}$ & 18 & 12 & Estômago & $*$ & $* *$ \\
\hline 22 & $\mathrm{~F}$ & 22 & 10 & Estômago/duodeno & $*$ & $* *$ \\
\hline 23 & $\mathrm{~F}$ & 31 & 8 & Duodeno & $*$ & $* *$ \\
\hline 24 & M & 27 & 7 & Duodeno & $*$ & $* *$ \\
\hline 25 & $\mathrm{~F}$ & 28 & 7 & Estômago/duodeno & $*$ & $* *$ \\
\hline 26 & $\mathrm{~F}$ & 28 & 6 & Duodeno & $*$ & $* *$ \\
\hline 27 & $\mathrm{M}$ & 28 & 2 & Estômago/duodeno & $*$ & $* *$ \\
\hline
\end{tabular}


Adenocarcinoma em papila duodenal foi encontrado no seguimento de uma doente, de 76 anos. Devido à idade avançada e co-morbidades, optou-se pela realização de duodenotomia, ressecção e reimplante da papila duodenal. Está em seguimento pósoperatório há 9 meses sem evidência de recidiva (Tabela 1).

\section{DISCUSSÃO}

A EDA tem possibilitado o rastreamento de pólipos gastroduodenais nessa população, detectando precocemente áreas de displasia e neoplasia duodenais, além de ser procedimento de baixa morbidade. A ocorrência de pólipos gastroduodenais em doentes com PAF em seguimento neste serviço foi elevada $(50,9 \%)$, ao passo que a ocorrência de adenocarcinoma duodenal foi de $1,9 \%$, número este semelhante ao da literatura ${ }^{(6,8,11,16)}$.

NUGEN et al. ${ }^{(10)}$ relataram $95 \%$ de ocorrência de pólipos duodenais e $5 \%$ de adenocarcinoma duodenal nessa população. CHURCH et al. ${ }^{(4)}$, observaram adenomas no trato gastrointestinal superior acima de $90 \%$, principalmente no duodeno. VASEN et al. ${ }^{(18)}$ descreveram adenomas duodenais em $90 \%$ dos pacientes com PAF, e adenocarcinoma duodenal em 3\% a 4\%. BULOW et al. ${ }^{(2}$ ), em análise de 368 casos com PAF, encontraram pólipos duodenais em número menor que a dos autores anteriores $(65 \%)$ e taxa cumulativa de incidência de câncer duodenal de 4,5\%.

TULCHINSKY et al. ${ }^{(17)}$ estudaram 50 doentes com PAF pósretocolectomia total, dos quais 41 tiveram seguimento endoscópico e, destes, 11 desenvolveram adenomas duodenais (26,8\%). Esses autores apresentaram taxa de aderência ao seguimento de $82 \%$, próxima a obtida neste estudo $(88,7 \%)$.

A causa de mortalidade dos doentes com PAF que foram submetidos a retocolectomia, tem mudado substancialmente, sendo o tumor duodenal e o tumor desmóide as causas mais freqüentes de óbito nesta população ${ }^{(9,17)}$.

SPIGELMAN et al. ${ }^{(16)}$ propuseram uma classificação para os pólipos duodenais de acordo com o número de pólipos encontrados, além do tamanho, histologia e grau de displasia. A cirurgia estaria indicada, segundo os autores, no estágio III e IV de Spigelman. Neste estudo não foi utilizada a classificação de Spigelman. No entanto a indicação de intervenção terapêutica nos casos de adenocarcinoma e adenomas com displasia de alto grau são indiscutíveis ${ }^{(3)}$.

A escolha da melhor opção terapêutica a realizar-se nestes casos pode, no entanto, não ser tão clara e irá depender das condições clínicas do doente e a localização dos pólipos ${ }^{(15)}$. A polipectomia endoscópica e a duodenotomia com polipectomia cirúrgica são condutas mais conservadoras que previnem o câncer temporariamente, com baixa morbidade, porém com alta taxa de recurrência dos pólipos ${ }^{(1,3,13)}$. Já a duodenectomia ou duodenopancreatectomia são condutas mais invasivas, "curativas", porém com alta taxa de morbimortalidade ${ }^{(3)}$.

\section{CONCLUSÕES}

Dentro dos limites metodológicos propostos para este estudo, com análise retrospectiva da casuística, o rastreamento endoscópico foi eficiente em detectar as lesões gastroduodenais e principalmente o caso de adenocarcinoma duodenal ainda em fase precoce, e também demonstrou boa aderência ao programa de acompanhamento pós-operatório. Estudos prospectivos, no entanto, são necessários para melhor avaliar a eficácia e necessidade desse acompanhamento e desenvolver estratégias preventivas e curativas efetivas.

Leal RF, Ayrizono MLS, Coy CSR, Callejas-Neto F, Fagundes JJ, Góes JRN. Gastroduodenal polyposis in patients with familiar adenomatous polyposis after rectocolectomy. Arq Gastroenterol. 2007;44(2):133-6.

ABSTRACT - Background - The extra colonic manifestations, like upper gastrointestinal tract polyps and duodenal cancer are disorders that affect long-term morbidity and mortality of patients with familial adenomatous polyposis, after rectocolectomy. Aim - To describe the frequency of those disorders in patients with familial adenomatous polyposis and to review efficacy of upper gastrointestinal endoscopic surveillance. Methods - Between 1984 and 2005 , 62 patients with familial adenomatous polyposis after rectocolectomy, were studied retrospectively, by Coloproctology Group, Medical Sciences Faculty, State University of Campinas, SP, Brazil. It was possible to analyze 53 patients (85,5\%) in this study. Results - Twenty seven (50,9\%) of 53 patients in follow-up had upper gastrointestinal polyps. Eight (15,4\%) had gastric adenomatous polyps, 14 (27\%), duodenal polyps and $5(9,6 \%)$ duodenal and gastric polyps. Two patients $(3,8 \%)$ had adenomatous duodenal polyps with severe dysplasia, and one (1,9\%) had adenocarcinoma of the duodenal papilla. Conclusions - The upper gastrointestinal endoscopic surveillance has importance and the aim is to detect as early as possible the occurrence of duodenal adenocarcinoma and upper gastrointestinal polyps with severe dysplasia.

HEADINGS - Adenomatous polyposis coli. Intestinal polyps. Colonic pouches. Proctocolectomy, restorative. 


\section{REFERÊNCIAS}

1. Alarcon FJ, Burke CA, Church JM, van Stolk RU. Familial adenomatous polyposis: efficacy of endoscopic and surgical treatment for advanced duodenal adenomas. Dis Colon Rectum. 1999;42:1533-6.

2. Bulow S, Bjork J, Christensen IJ, Fausa O, Jarvinen H, Moesgaard F, Vasen HFA. Duodenal adenomatosis in familial adenomatous polyposis. Gut. 2004;53:381-6.

3. Cappel WHVN, Jarvinen HJ, Bjork J, Berk T, Griffioen G, Vasen HFA. Worldwide survey among polyposis registries of surgical management of severe duodenal adenomatosis in familial adenomatous polyposis. Br J Surg. 2003;90:705-10.

4. Church JM, McGannon E, Hull-Boiner S, Sivak MV, Van Stolk R, Jagelman DG Gastroduodenal polyps in patients with familial adenomatous polyposis. Dis Colon Rectum. 1992;35:1170-3.

5. Domizio P, Talbot IC, Spilgeman AD, Williams CB, Phillips RK. Upper gastrointestina pathology in familial adenomatous polyposis: results from a prospective study of 102 patients. J Clin Pathol. 1990;43:738-43.

6. Groves CJ, Saunders BP, Spilgeman AD, Phillips RKS. Duodenal cancer in patients with familial adenomatous polyposis (FAP): results of a 10 year prospective study. Gut. 2002;50:636-41.

7. Hampel H, Peltomaki P. Hereditary colorectal cancer: risk assessment and management. Clin Genet. 2000;58:89-97.

8. Jagelman DG, DeCosse, Bussey HJ. Upper gastrointestinal cancer in familial adenomatous polyposis. Lancet. 1988;1:1149-51.

9. King JE, Dozois RR, Lindor NM, Ahlquist DA. Care of patients and their families with familial adenomatous polyposis. Mayo Clin Proc. 2000;75:57-67.
10. Nugen KP, Spilgeman AD, Phillips RK. Life expectancy after colectomy and ileorectal anastomosis for familial adenomatous polyposis. Dis Colon Rectum. 1993;36:1059-62.

11. Offerhaus GJ, Giardello FM, Krush AJ, Booker SV, Tersmette AC, Kelley NC. The risk of upper gastrointestinal cancer in familial adenomatous polyposis. Gastroenterology. 1992;102:1980-2.

12. Pauli RM, Pauli ME, Hall JG. Gardner syndrome and periampullary malignancy. Am J Med Genet. 1980;6:205-19.

13. Penna C, Bataille N, Balladur P, Tiret E, Parc R. Surgical treatment of severe duodenal polyposis in familial adenomatous polyposis. Br J Surg. 1998;85:665-8.

14. Phillips RKS, Spilgeman AD, Thomson JPS, editors. Familial adenomatous polyposis and others polyposis syndromes. London: Edward Arnold; 1994.

15. Saurin JC, Gutknecht C, Napoleon B, Chavaillon A, Ecochard R, Scoazec JY, Ponchon T, Chayvialle JA. Surveillance of duodenal adenomas in familial adenomatous polyposis reveals high cumulative risk of advanced disease. J Clin Oncol. 2004;22:493-8.

16. Spigelman AD, Williams CB, Talbot IC, Domizio P, Phillips RK. Upper gastrointestinal cancer in patients with familial adenomatous polyposis. Lancet. 1989;2:783-5.

17. Tulchinsky H, Keidar A, Strul H, Goldman G, Klausner J, Rabau M. Extracolonic manifestations of familial adenomatous polyposis after proctocolectomy. Arch Surg. 2005; 140:159-64

18. Vasen HF, Bulow S, Myrhoj T, Mathus-Vliegen L, Griffioen G, Buskens E. Decision analysis in the management of duodenal adenomatosis in familial adenomatous polyposis. Gut. 1997;40:716-9. 